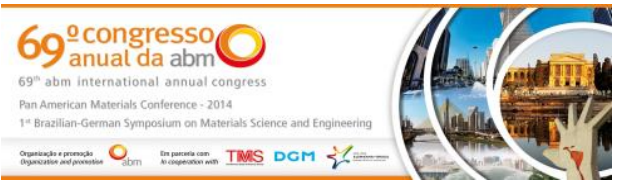

Tema: Diagramas de fases e transformações de fases

\title{
CARACTERIZAÇÃO DAS FASES DE AÇOS SUPERDUPLEX ATRAVÉS DA SIMULAÇÃO TERMODINÂMICA- COMPUTACIONAL*
}

\author{
Rodrigo Afonso Hatwig ${ }^{1}$ \\ Nestor Cezar Heck ${ }^{2}$ \\ Alexandre da Silva Rocha ${ }^{3}$ \\ Luciano Aparecido Kempski ${ }^{4}$
}

\section{Resumo}

Os aços inoxidáveis duplex (DSS) e superduplex (SDSS) são ligas que se caracterizam por apresentar uma microestrutura composta por frações volumétricas similares das fases ferrita e austenita. Dependendo da composição química e do tratamento termomecânico aplicado, a microestrutura dos aços inoxidáveis DSS e SDSS poderá apresentar, além das fases ferrita e austenita, fases intermetálicas deletérias. Neste trabalho, um estudo termodinâmico-computacional foi realizado com o objetivo de acompanhar a evolução das fases de aços superduplex no intervalo de temperaturas entre $300^{\circ} \mathrm{C}$ e $1.200^{\circ} \mathrm{C}$. Para tanto, fez-se uso do software de simulação termodinâmica FactSage v. 6.4. Os resultados descrevem de forma eficaz as fases principais e secundárias em função da temperatura e composição química.

Palavras-chave: Aços inoxidáveis duplex; Simulação termodinâmica; Transformação de fases; Fases intermetálicas nocivas;

\section{CHARACTERIZATION OF THE PHASES OF SUPERDUPLEX STEELS THROUGH COMPUTER SIMULATION THERMODYNAMICS}

\section{Abstract}

The duplex (DSS) and superduplex (SDSS) stainless steel alloys are characterized by having a microstructure consisting of similar volume fractions of ferrite and austenite phases. Depending on the chemical composition and thermomechanical treatment applied to the microstructure of stainless steel SDSS and DSS may display, in addition to austenite and ferrite phases, harmful intermetallic phases. In this work, a thermodynamic-computational study was carried out in order to monitor the phase evolution in the steel in the temperature range between $300^{\circ} \mathrm{C}$ and $1,200^{\circ} \mathrm{C}$. In order to effect this, the thermodynamic simulation software FactSage $\vee 6.4$ was used. The results describe effectively the primary and secondary phases as a function of temperature and chemical composition.

Keywords: Duplex stainless steels; Thermodynamic simulation; Transformation of phases; Deleterious intermetallic phases.

1 Engenheiro Metalurgista, aluno do Programa de Pós-Graduação em Engenharia de Minas, Metalúrgica e de Materiais (PPGE3M), Universidade Federal do Rio Grande do Sul (UFRGS), Porto Alegre, RS, Brasil.

2 Engenheiro Metalurgista, Dr., Professor, Núcleo de Termodinâmica Computacional para a Metalurgia (NTCm), UFRGS, Porto Alegre, RS, Brasil.

3 Engenheiro Mecânico, Dr., Professor, Laboratório de Transformação Mecânica (LdTM), UFRGS, Porto Alegre, RS, Brasil.

4 Engenheiro Mecânico, MSc., Professor, IFRS - Campus Erechim, doutorando do PPGE3M, UFRGS, Porto Alegre, RS, Brasil

\footnotetext{
* Contribuição técnica ao $69^{\circ}$ Congresso Anual da ABM - Internacional e ao 14ํㅡㄹ ENEMET - Encontro Nacional de Estudantes de Engenharia Metalúrgica, de Materiais e de Minas, 21 a 25 de julho de 2014, São Paulo, SP, Brasil.
} 


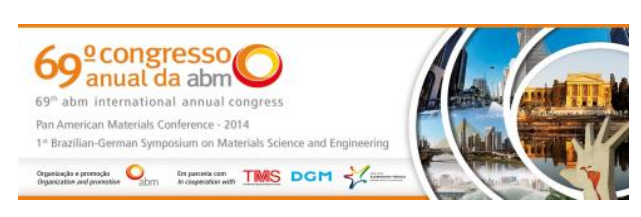

\section{INTRODUÇÃO}

Aços inoxidáveis duplex (DSS) e superduplex (SDSS) são caracterizados por uma estrutura de duas fases, compreendendo uma mistura de grãos de ferrita e austenita em frações volumétricas aproximadamente iguais [1]. Esta microestrutura, quando isenta de partículas secundárias, oferece uma combinação de alta dureza, resistência à corrosão, boa soldabilidade e alta resistência mecânica [2].

Entretanto, dependendo da composição química e do tratamento termomecânico aplicado, a microestrutura dos aços inoxidáveis DSS e SDSS poderá apresentar, além das fases ferrita e austenita, várias fases intermetálicas deletérias.

As principais propriedades apresentadas pelos aços inoxidáveis duplex e superduplex resultam de uma combinação das melhores características das suas duas fases: (i) a austenita, que contribui para a resistência ao impacto e, (ii) a ferrita, que melhora a resistência mecânica e a soldabilidade.

Além da ferrita e da austenita uma grande variedade de fases secundárias 'indesejáveis' podem se formar no intervalo de temperaturas de $300^{\circ} \mathrm{C}-1000^{\circ} \mathrm{C}$ nos SDSS durante o envelhecimento isotérmico ou um tratamento térmico incorreto. Dentre outras, podem ser observadas as seguintes fases: fase $\sigma, \mathrm{Cr}_{2} \mathrm{~N}, \mathrm{CrN}$, austenita secundária, fase $\chi$, fase $\pi$, fase $R, M_{7} C_{3}, M_{23} C_{6}, C u$, e fase $\tau$; isto se deve, essencialmente, à instabilidade da ferrita.

Das fases citadas acima, a fase $\sigma$ é a mais importante em virtude da sua influência sobre a dureza e a resistência à corrosão. Um resumo contendo algumas características destas fases secundárias pode ser encontrado na literatura [3].

A principal diferença entre os aços duplex e superduplex está nos teores de $\mathrm{Cr}$ e $\mathrm{N}$, que são superiores nos SDSS. Contudo, o critério de classificação empregado na distinção entre os aços inoxidáveis duplex e os superduplex está no parâmetro conhecido como 'resistência ao pite equivalente' (pitting resistance equivalent, PRE), cujo valor é definido pela Equação 1 [4].

$$
\mathrm{PREN}_{\mathrm{N}}=\% \mathrm{Cr}+3,3 \% \mathrm{Mo}+16 \% \mathrm{~N}
$$

Em particular, os valores de PREN nos SDSS são superiores a 40, o que indica uma maior resistência à corrosão [1]. Boas propriedades mecânicas e elevada resistência ao pite equivalente estão associados a altos teores de $\mathrm{Cr}$, Mo e $\mathrm{N}$ e sua fina dispersão na microestrutura. Por estes motivos, os aços inoxidáveis superduplex são amplamente utilizados em plataformas 'offshore'.

Neste trabalho, um estudo sobre as transformações das fases de um aço superduplex, incluindo a avaliação das fases secundárias, será realizado. Esta abordagem é de fundamental importância, uma vez que o controle dos parâmetros de solidificação e tratamento térmico empregados nos aços inoxidáveis superduplex está diretamente ligado à maximização de suas propriedades (mecânicas e de resistência à corrosão). Este estudo, que tem por objetivo compreender a evolução dos precipitados durante o processamento dos aços SDSS, fica, dessa forma, justificado.

\section{MATERIAIS E MÉTODOS}

Os diagramas de fase ternários Fe-Cr-Ni e a caracterização das fases dos sistemas Fe-Cr-Ni e Fe-Cr-Ni-Mo-N-C foram produzidos com o auxílio do software de simulação termodinâmica FactSage v. 6.4.

\footnotetext{
* Contribuição técnica ao $69^{\circ}$ Congresso Anual da ABM - Internacional e ao 14ํㅡㄹ ENEMET - Encontro Nacional de Estudantes de Engenharia Metalúrgica, de Materiais e de Minas, 21 a 25 de julho de 2014, São Paulo, SP, Brasil.
} 


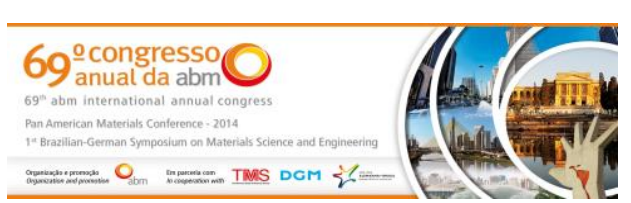

Os dados termodinâmicos estão contidos nos bancos de dados (i) FSstel (FSstel53base.cdb) e, (ii) FactPS (FS53base.cdb).

A composição química foi escolhida levando-se em consideração a composição química típica de um tipo de aço inoxidável superduplex. Além disso, os elementos escolhidos foram: $\mathrm{Fe}, \mathrm{Cr}, \mathrm{Ni}, \mathrm{Mo}, \mathrm{N}, \mathrm{C}$, pois eles fazem parte da composição química das partículas secundárias com maior ocorrência.

O trabalho apresenta resultados com temperaturas no intervalo de 300 a $1200^{\circ} \mathrm{C}$.

\section{RESULTADOS E DISCUSSÃO}

\subsection{Sistema Fe-Cr-Ni}

\subsubsection{Diagramas de fase isotérmicos}

Inicialmente foi realizado um estudo considerando o sistema ternário simples Fe-Cr-Ni. Este sistema é de grande importância, pois permite a compreensão global da evolução das fases e fornece uma base para a compreensão da microestrutura dos aços inoxidáveis.

Em [5], está apresentada uma visão tridimensional do sistema ternário Fe-Cr-Ni destacando o campo da fase sigma. Nesta representação, podem ser visualizadas apenas as fases principais: ferrita, austenita e sigma.

Seções horizontais, ou cortes isotérmicos, foram realizados no diagrama apresentado por [5], com o intuito de tornar a observação das fases citadas mais acurada e de facilitar o entendimento da evolução do campo da fase sigma em função da temperatura.

As Figuras 1-6 mostram, a seguir, representações das seções isotérmicas do diagrama ternário Fe-Cr-Ni em diferentes temperaturas.

\subsubsection{Simulação do resfriamento do sistema ternário Fe-Cr-Ni no estado de equilíbrio.}

Uma simulação termodinâmica mostrando as fases em equilíbrio em função da temperatura foi feita simulando o resfriamento do sistema ternário $\mathrm{Fe}-\mathrm{Cr}-\mathrm{Ni}$.

Nestas simulações foi utilizada uma composição simplificada dos aços inoxidáveis superduplex, Tabela 1.

Ela reproduz basicamente os teores de $\mathrm{Cr}$ e $\mathrm{Ni}$ encontrados em aços do tipo escolhido para este trabalho.

Tabela 1. Composição química do sistema ternário Fe-Cr-Ni

\begin{tabular}{|c|c|c|c|}
\hline $\begin{array}{c}\text { Elemento } \\
\text { químico }\end{array}$ & $\mathrm{Fe}$ & $\mathrm{Cr}$ & $\mathrm{Ni}$ \\
\hline $\begin{array}{c}\text { Porcentagem } \\
\text { ponderal, \% }\end{array}$ & 68 & 25 & 7 \\
\hline
\end{tabular}

A Figura 7 mostra o resultado do cálculo realizado em termos da porcentagem ponderal das fases em função da temperatura, levando-se em consideração a composição química da Tabela 1.

\footnotetext{
* Contribuição técnica ao $69^{\circ}$ Congresso Anual da ABM - Internacional e ao 14ํㅡㄹ ENEMET - Encontro Nacional de Estudantes de Engenharia Metalúrgica, de Materiais e de Minas, 21 a 25 de julho de 2014, São Paulo, SP, Brasil.
} 

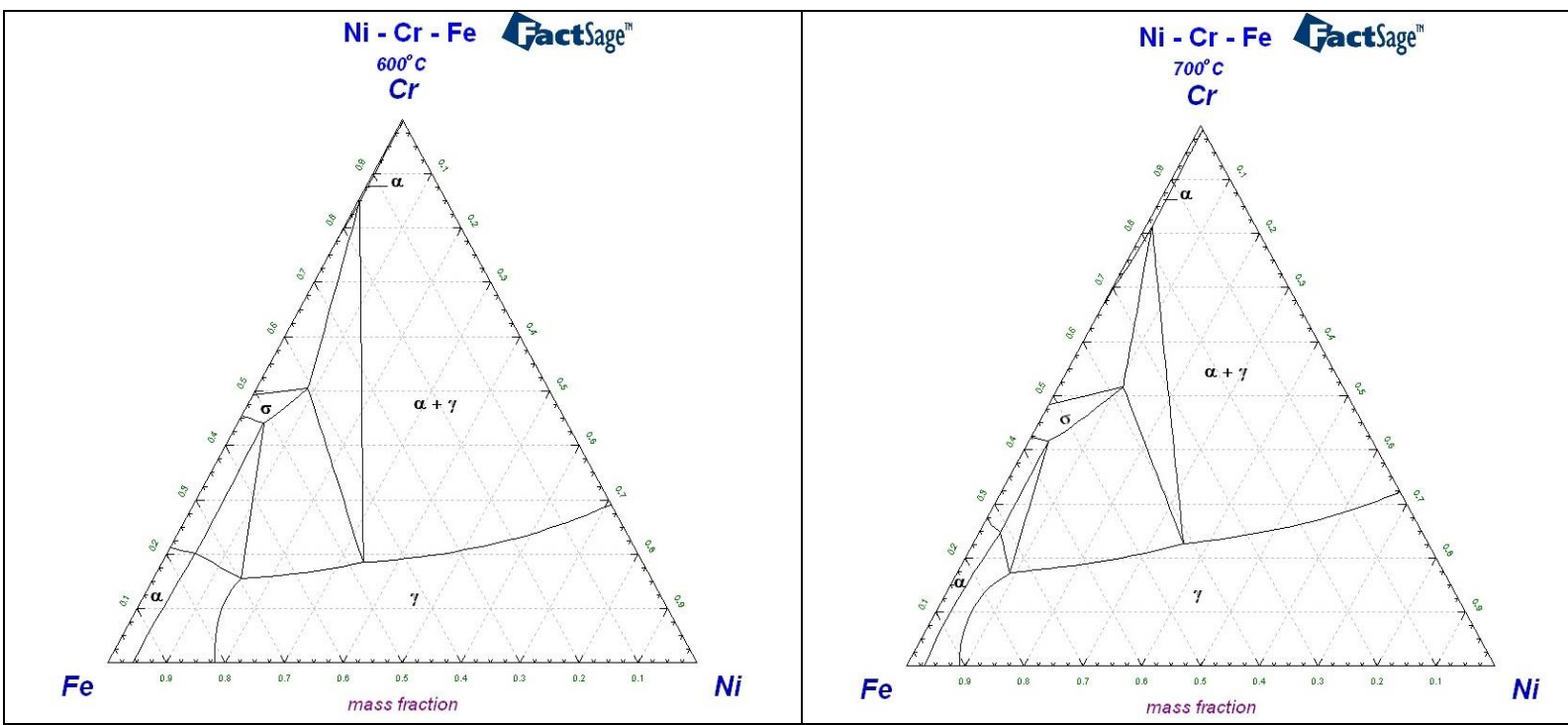

Figura 1. Diagrama $\mathrm{Fe}-\mathrm{Cr}-\mathrm{Ni}$; temperatura $600^{\circ} \mathrm{C}$.

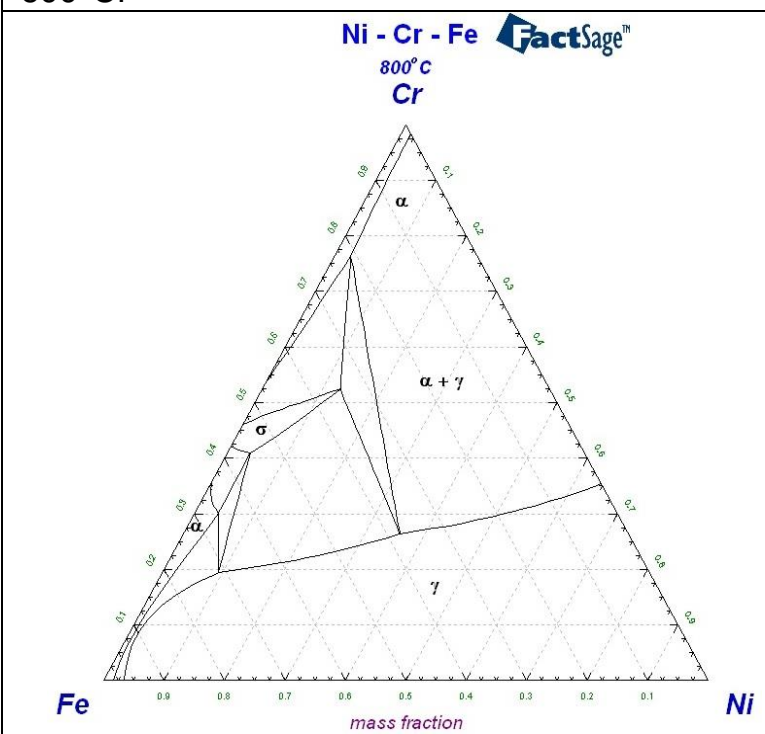
$700^{\circ} \mathrm{C}$.

Figura 3. Diagrama $\mathrm{Fe}-\mathrm{Cr}-\mathrm{Ni}$; temperatura $800^{\circ} \mathrm{C}$.

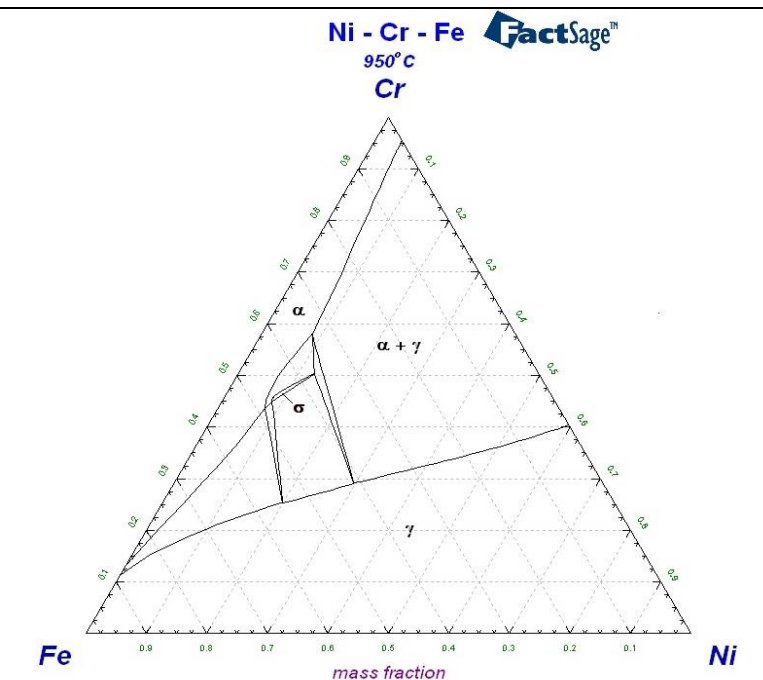

Figura 5. Diagrama $\mathrm{Fe}-\mathrm{Cr}-\mathrm{Ni}$; temperatura $950^{\circ} \mathrm{C}$

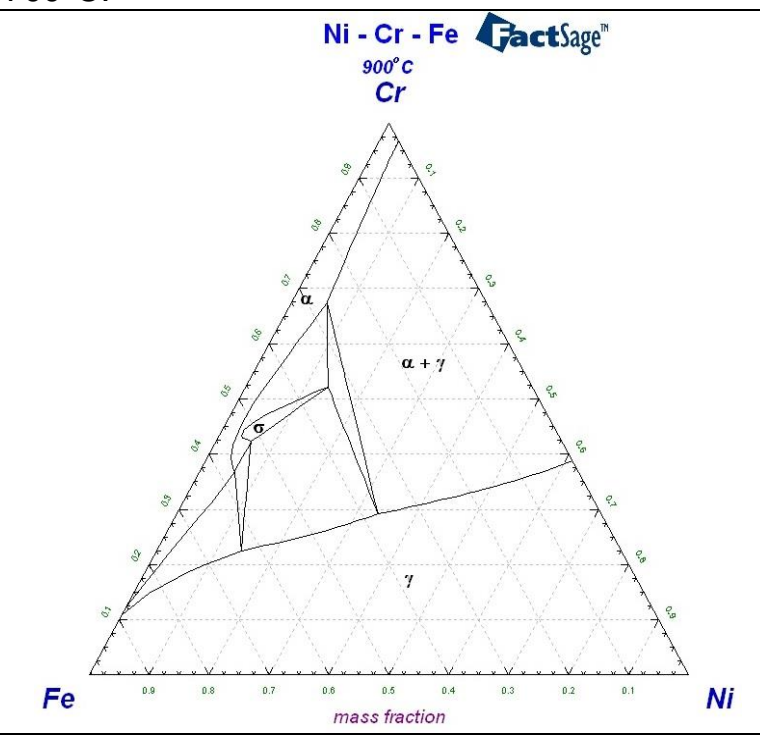

Figura 4. Diagrama $\mathrm{Fe}-\mathrm{Cr}-\mathrm{Ni}$; temperatura $900^{\circ} \mathrm{C}$.

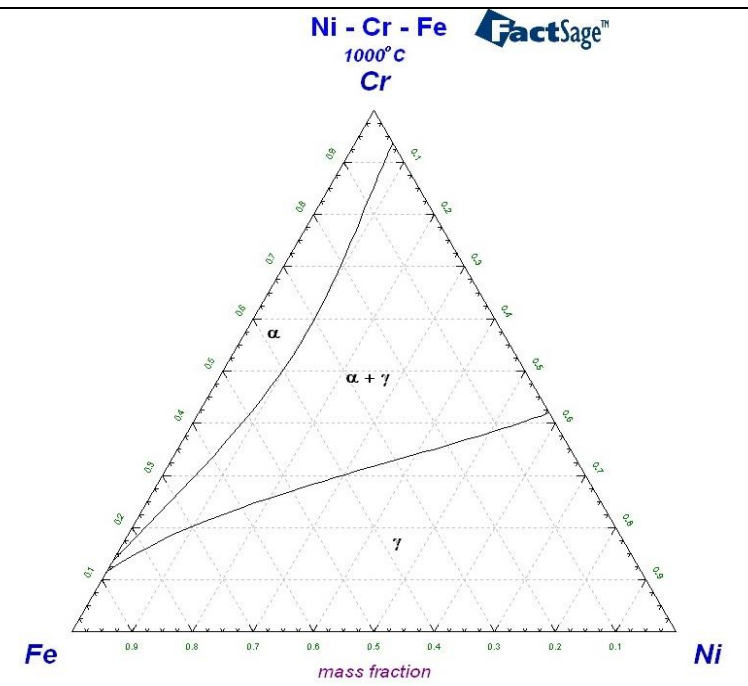

Figura 6. Diagrama $\mathrm{Fe}-\mathrm{Cr}-\mathrm{Ni}$; temperatura $1000^{\circ} \mathrm{C}$.

* Contribuição técnica ao $69^{\circ}$ Congresso Anual da ABM - Internacional e ao 14ํㅡㄹ ENEMET - Encontro Nacional de Estudantes de Engenharia Metalúrgica, de Materiais e de Minas, 21 a 25 de julho de 2014, São Paulo, SP, Brasil. 

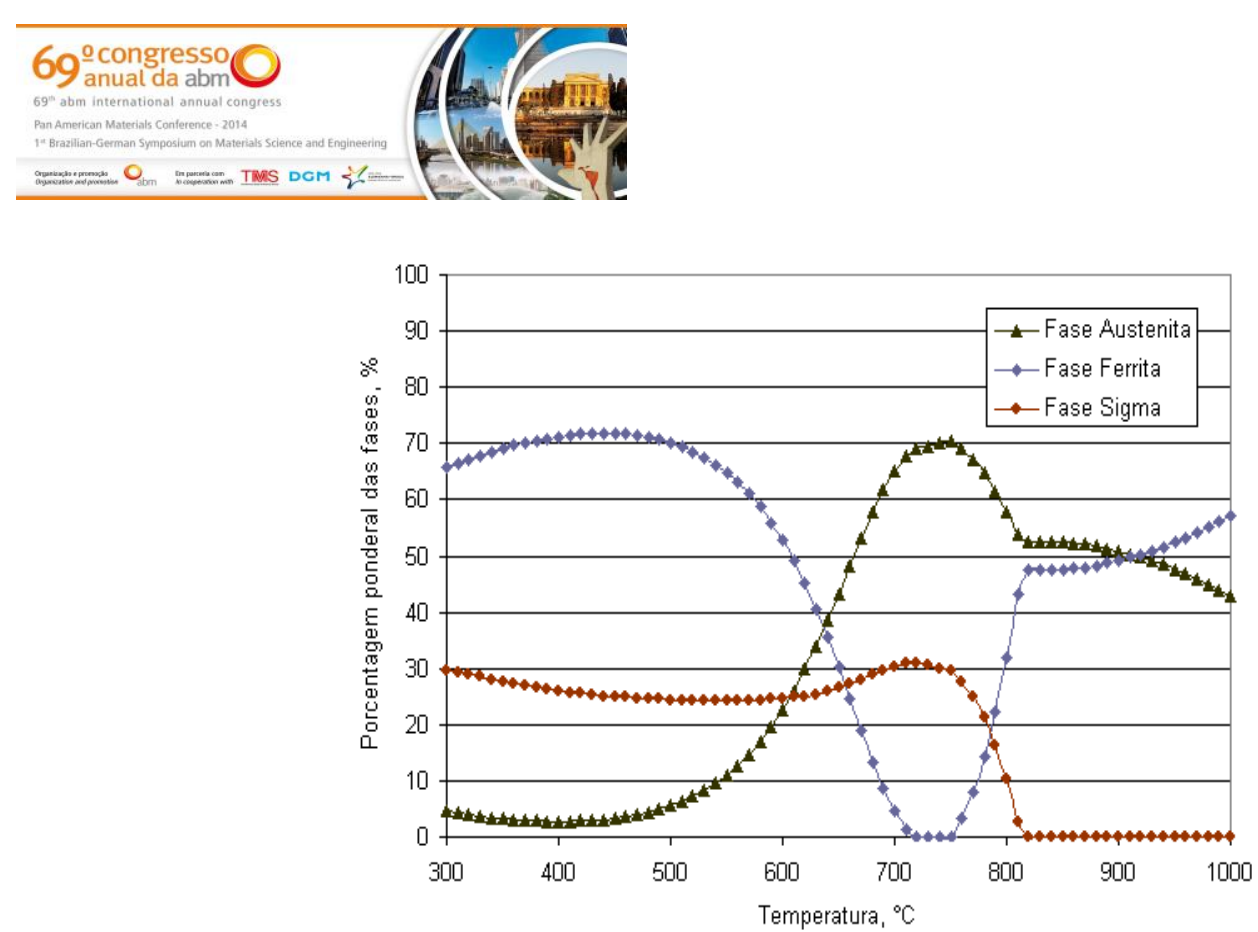

Figura 7. Evolução das fases no sistema ternário Fe-Cr-Ni em função da temperatura

Verifica-se, na Figura 7, a ocorrência de um ponto de equilíbrio entre as massas das fases ferrita e austenita na temperatura de $910^{\circ} \mathrm{C}$. Na indústria usa-se tomar a temperatura onde os volumes destas fases são idênticos. Este ponto é de grande importância, pois nesta temperatura os aços inoxidáveis DSS e SDSS apresentam as melhores propriedades mecânicas e de resistência à corrosão.

\subsubsection{O Diagrama de Schaeffler}

Existem nos aços inoxidáveis dois grupos de elementos de liga: (i) os que estabilizam a ferrita, tais como o $\mathrm{Cr}, \mathrm{Si}, \mathrm{Mo}, \mathrm{Ti}$ e $\mathrm{Nb}$ e, (ii) os que estabilizam a austenita, como o $\mathrm{Ni}, \mathrm{C}, \mathrm{N}$ e $\mathrm{Mn}$. Com a finalidade de consolidar seus efeitos, os elementos de liga com características semelhantes foram agrupados e introduziu-se, então, o conceito de cromo e níquel equivalentes conforme representado pelas Equações 2 e 3.

$$
\begin{aligned}
& \mathrm{Cr}_{\text {eq }}=\% \mathrm{Cr}+\% \mathrm{Mo}+1.5 \% \mathrm{Si}+0.5 \% \mathrm{Nb} \\
& \mathrm{Ni} \text { eq }=\% \mathrm{Ni}+0.5 \% \mathrm{Mn}+30(\% \mathrm{C}+\% \mathrm{~N})
\end{aligned}
$$

Realizando-se o cálculo do $\mathrm{Cr}_{\text {eq }}$ e $\mathrm{Ni}_{\text {eq }}$, segundo as equações tradicionais 2 e $3 \mathrm{com}$ a composição química do aço inoxidável superduplex sugerida adiante, encontra-se os valores $\mathrm{Cr}_{\text {eq }}=28,5$ e $\mathrm{Ni}_{\text {eq }}=15,4$.

Visualizando-se, então, os valores obtidos no diagrama de Schaeffler, lê-se um percentual de ferrita no aço entre 10 e $20 \%$.

Outras equações descrevendo $\mathrm{Cr}_{\text {eq }}$ e Nieq são citadas na literatura [4]:

$$
\begin{aligned}
& \text { Creq. }=(\%) \mathrm{Cr}+[(1,5) \cdot(\%) \mathrm{Si}]+[(1,4) \cdot(\%) \mathrm{Mo}]+(\%) \mathrm{Nb}-4,99 \\
& \text { Nieq. }=(\%) \mathrm{Ni}+[(30) .(\%) \mathrm{C}]+[(0,5) \cdot(\%) \mathrm{Mn}]+[26(\%) \mathrm{N}-0,02)]+2,77
\end{aligned}
$$

\subsection{Sistema Fe-Cr-Ni-Mo-N-C}

A partir deste ponto do estudo foi considerando o sistema ternário mais complexo Fe-Cr-Ni-Mo-N-C. Este sistema permite a compreensão das fases secundárias, que podem ser relacionadas com efeitos negativos sobre a resistência mecânica dos aços inoxidáveis.

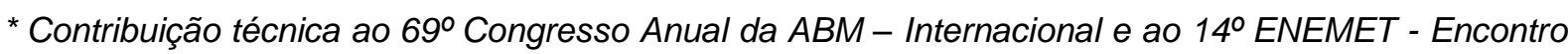
Nacional de Estudantes de Engenharia Metalúrgica, de Materiais e de Minas, 21 a 25 de julho de 2014, São Paulo, SP, Brasil.
} 


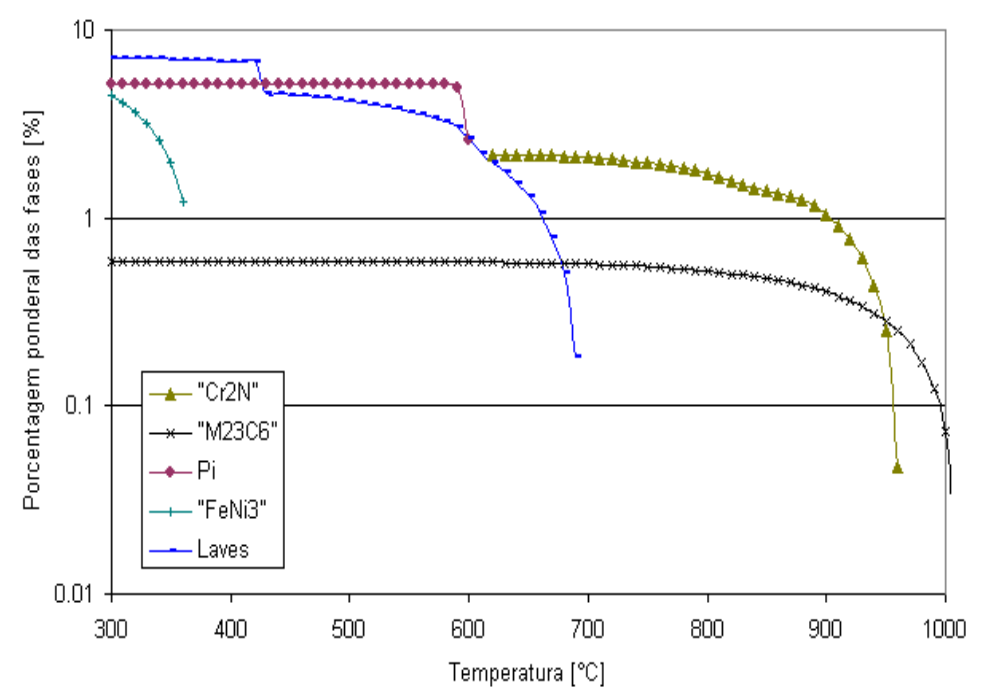

Figura 9. Evolução das fases secundárias do sistema Fe-Cr-Ni-Mo-N-C

As fases secundárias normalmente são representadas como compostos estequiométricos (intermetálicos). Na presente análise, contudo, estas fases são soluções sólidas - o que demonstra a grande complexidade dos bancos de dados presentes no aplicativo de termodinâmica computacional empregado, Tabela 3.

Tabela 3. Composição química das fases secundárias do sistema Fe-Cr-Ni-Mo-N-C em algumas temperaturas escolhidas.

\begin{tabular}{|l|l|c|c|c|c|c|c|c|}
\hline & Fase & $\begin{array}{c}\text { Estrutura } \\
\text { cristalina }\end{array}$ & $\mathrm{Fe}$ & $\mathrm{Ni}$ & $\mathrm{Cr}$ & $\mathrm{Mo}$ & $\mathrm{N}$ & $\mathrm{C}$ \\
\hline \multirow{3}{*}{$300^{\circ} \mathrm{C}$} & Laves & hcp & 37 & & 16 & 47 & & \\
\cline { 2 - 9 } & 'M23C6' & cfc & 4 & & 71 & 21 & & 5 \\
\cline { 2 - 9 } & $\mathrm{Pi}$ & Cúbica & & 37 & 58 & & 5 & \\
\cline { 2 - 9 } & 'FeNi3' & & 32 & 68 & & & & \\
\hline \multirow{3}{*}{$500^{\circ} \mathrm{C}$} & Laves & hcp & 38 & & 16 & 47 & & \\
\cline { 2 - 9 } & 'M23C6' & cfc & 8 & & 66 & 20 & & 5 \\
\cline { 2 - 9 } & $\mathrm{Pi}$ & Cúbica & 1 & 36 & 58 & & 5 & \\
\hline \multirow{2}{*}{$700^{\circ} \mathrm{C}$} & 'Cr2N' & hcp & & & 83 & 6 & 11 & \\
\cline { 2 - 9 } & 'M23C6' & cfc & 14 & & 61 & 2 & & 5 \\
\hline
\end{tabular}

\section{CONCLUSÕES}

Considerando-se as composições simplificadas das duas representações de aços inoxidáveis superduplex empregados no presente estudo, pode-se concluir que há uma diferença significativa entre as massas relativas das fases austenita, ferrita e sigma em função da temperatura.

A previsão da formação de fases secundárias nestes aços, em função de processos de fabricação ou tratamentos térmicos, pôde ser confirmada no presente trabalho; além da fase sigma, são dignas de nota as fases Laves, $\mathrm{Pi}(\pi)$, "Cr $2 \mathrm{~N}$ " e $\mathrm{M}_{23} \mathrm{C}_{6}$.

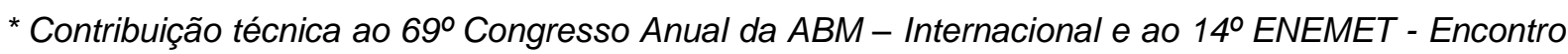
Nacional de Estudantes de Engenharia Metalúrgica, de Materiais e de Minas, 21 a 25 de julho de 2014, São Paulo, SP, Brasil.
} 


\section{Agradecimentos}

O autor Rodrigo Afonso Hatwig agradece à Comissão de Aperfeiçoamento de Pessoal de Nível Superior, CAPES, pela bolsa de estudos recebida.

\section{REFERÊNCIAS}

1 Weber J. Materials for Seawater Pumps and Related Systems. Internal Technical Publication. Winterthur: Sulzer Brothers Limited; 1986. p.1-12.

2 Bonollo F, Tiziani AP. Ferro: Duplex Stainless Steels. 1를 ed. Iris Alvarez Armas, Degallaix-Moreuil S. Great Britain: John Wiley \& Sons; 2009. p.141-155.

3 Lo KH, Shek CH, Lai JKL. Recent developments in stainless steels. Materials Science and Engineering R. 2009;65:39-104.

4 ASTM International. ASTM Standard A 890/A 890M-91: Ferrous Castings; Ferralloys, Annual Book of ASTM Standards, vol. 1-2. West Conshohocken; 1999.

5 Pardal JM, Tavares SSM, Cindra FMP, Souza JA, Corte RRA, Abreu HFG. Influence of the grain size on deleterious phase precipitation in superduplex stainless steel UNS S32750. Materials Characterization. 2009;60:165-172.

* Contribuição técnica ao $69^{\circ}$ Congresso Anual da ABM - Internacional e ao 14ํㅡㄹ ENEMET - Encontro Nacional de Estudantes de Engenharia Metalúrgica, de Materiais e de Minas, 21 a 25 de julho de 2014, São Paulo, SP, Brasil. 